\title{
AN EXTENSION OF RATIONAL CONTRACTIVE CONDITION IN PARTIALLY ORDERED RECTANGULAR METRIC SPACES
}

\author{
MEHMET KIR, MEHMET ALI AKTURK, HEMEN DUTTA, AND ESRA YOLACAN
}

Received 19 October, 2016

\begin{abstract}
In this paper, we discuss the concept of partially ordered rectangular metric spaces using the notation $(X, \preceq, d)$. Also, we give a generalized contractive condition in the context of partially ordered rectangular metric space for all comparable pair of $X$. Our results extend and generalize the well-known results of [13], [1], [14] and [11].
\end{abstract}

2010 Mathematics Subject Classification: 41A65; 41A15; 47H09; 47H10; 54H25

Keywords: fixed point, rectangular metric, generalized metric spaces

\section{INTRODUCTION}

Banach contraction principle proved by mathematician S. Banach was published in 1922. This principle is one of the most useful tools in the study of nonlinear equations. This contraction mapping principle has been generalized in many directions ([3], [7], [10], [17], [19], [5]).

In 1973, Geraghty [7] introduced the following generalization of Banach's contraction principle that known Geraghty type contraction.

Let $(X, d)$ be a complete metric space and let $f: X \rightarrow X$ be map. Suppose there exists $\beta \in \vartheta$ such that for each $x, y \in X$

$$
d(f x, f y) \leq \beta(d(x, y)) d(x, y)
$$

where $\vartheta=\left\{\beta: \beta\left(t_{n}\right) \rightarrow 1\right.$ implies $\left.t_{n} \rightarrow 0\right\}$. Then $f$ has a unique fixed point $z \in X$ and $\left\{f^{n} x\right\}$ converges to $z$, for each $x \in X$.

In 1977, Jaggi [10] proved the following theorem satisfying a rational type contractive inequality.

Let $(X, d)$ be a complete metric space and let $f: X \rightarrow X$ be a continuous mapping such that

$$
d(f x, f y) \leq \alpha \frac{d(x, f x) d(y, f y)}{d(x, y)}+\beta d(x, y)
$$

for all distinct points $x, y \in X$ where $\alpha, \beta \in[0,1)$ with $\alpha+\beta<1$. Then $f$ has a unique fixed point. 
In 2000, Branciari introduced the notation of a generalized metric spaces. A generalized metric is a semi-metric which does not satisfy the triangle inequality but satisfies a weaker condition called quadrilateral inequality.

A rectangular metric space needs not to

(1) have a continuous rectangular metric

(2) have a convergent sequence as a Cauchy sequence.

Branciari also established an analogue of Banach's fixed point theorem on rectangular metric spaces. Extension of this analogue has been made in many directions ([4], [18], [2], [12]).

In 2004, Ran and Reurings [16] established an analogue of Banach's fixed point theorem in partially ordered set.

Theorem 1. Let $(X, \preceq)$ be a partially ordered set endowed with a metric $d$ and $(X, d)$ be a complete metric space. Furthermore, $X$ is such that

$$
\text { every pair } x, y \in X \text { has a lower bound and an upper bound. }
$$

If $f: X \rightarrow X$ is a continuous, monotone (i.e., either order-preserving or orderreversing) map from $X$ into $X$ such that

$$
\begin{gathered}
\exists 0<c<1: d(f x, f y) \leq c d(x, y), x \succeq y, \\
\exists x_{0} \in X: x_{0} \preceq f x_{0} \text { or } x_{0} \succeq f x_{0}, \\
\text { then } f \text { has a unique fixed point } \bar{x} \text {. Moreover, for every } x \in X \lim _{n \rightarrow \infty} f^{n} x=\bar{x} .
\end{gathered}
$$

One see that the difference of this theorem than the Banach contraction principle is that contractive inequality (1.2) satisfies only for comparable pair of the $(X, \preceq)$.

In 2005, Nieto and López [15] extended some results to study a problem of ordinary differential equations. Also, authors introduced the following concept to give some alternative conditions for the existence and uniqueness of the fixed point.

$$
\begin{aligned}
& \text { "if any nondecreasing sequence }\left\{x_{n}\right\} \text { in } X \text { converges to } z \text {, then } x_{n} \preceq z \\
& \qquad \text { for all } n \geq 0 \text { " } \\
& \text { "every pair of elements has a lower bound or an upper bound" }
\end{aligned}
$$

$$
\text { " for every } x, y \in X \text {; there exists } z \in X \text { which is comparable to } x \text { and } y " \text {. }
$$

According to Nieto and López [15]:

(1) The mapping defined as $f: X \rightarrow X$ needs not continuity if $(X, \preceq)$ has the property (1.3). 
(2) The partially ordered set $(X, \preceq)$ needs not the property (1.1). It is sufficient to consider that $X$ has the property (1.4).

(3) The property (1.4) is equivalent to the property (1.5).

Remark 1 ([15]). considered the map $f: X \rightarrow X$ as nondecreasing, but Ran and Reurings [16] studied as a monotone. In the other words, according to Ran and Reurings [16] it is sufficient to consider $f$ as monotone, that is, $f$ may be nondecreasing or nonincreasing.

Papers [8], [9], [13], [1], [14] and [11] were particularly focus on the following three points:

(1) The mapping defined by $f: X \rightarrow X$ is nondecreasing and continuous or $f$ is nondecreasing and the set $(X, \preceq)$ has the property (1.3).

(2) It is taken the assumption "if there exists $x_{0} \in X$ such that $x_{0} \preceq f x_{0}$ ".

(3) In order to guarantee the uniqueness of the fixed point, they needs the property (1.5).

In this paper we aim to

(1) introduce the concept of partially ordered rectangular metric space $(X, \preceq, d)$,

(2) give a generalized rational contractive condition that involve all results of the papers [1]-[5] and [7]-[19] without need some restrictions given in the above.

(3) Furthermore, we take the mapping defined by $f: X \rightarrow X$ is monotone and continuous or $f$ is monotone and the set $(X, \preceq)$ is regular.

We shall also discuss some results from [1]-[5] and [7]-[19] after giving the proof of our main results.

\section{MATHEMATICAL PRELIMINARIES}

Now, we recall some notations and remarks which are needed in the sequel.

Definition 1 ([4]). Let $X$ be a nonempty set and let $d: X \times X \rightarrow[0, \infty)$ satisfy the following conditions for all $x, y \in X$ and all distinct $u, v \in X$ each of them different from $x$ and $y$.

RM1) $d(x, y)=0$ if and only if $x=y$,

RM2) $d(x, y)=d(y, x)$,

$\mathrm{RM} 3) d(x, y) \leq d(x, u)+d(u, v)+d(v, y)$ ( quadrilateral inequality).

Then the function $d$ is called a rectangular metric and the pair $(X, d)$ is called a rectangular metric spaces (for short RMS).

In literature, generalized metric spaces (or in short g.m.s) instead of for rectangular metric spaces (in short RMS) is used by some researchers.

Definition $2([4])$. Let $(X, d)$ a rectangular metric spaces and $\left\{x_{n}\right\}$ be sequence in $X$. 
(1) $\left\{x_{n}\right\}$ is called g.m.s convergent to a limit $x$ if and only if $d\left(x_{n}, x\right) \rightarrow 0$ as $n \rightarrow \infty$,

(2) $\left\{x_{n}\right\}$ is called g.m.s Cauchy sequence if and only if for every $\epsilon>0$ there exists positive integer $N(\epsilon)$ such that $d\left(x_{n}, x_{m}\right)<\epsilon$ for all $n>m>N(\epsilon)$,

(3) A rectangular metric spaces $(X, d)$ is called complete if every g.m.s Cauchy sequence is g.m.s convergent.

Definition 3. Let $(X, \preceq, d)$ be a partially ordered rectangular metric space. $X$ is called regular if it has the following properties:

if a nondecreasing sequence $\left\{x_{n}\right\} \rightarrow x$ in $(X, d)$, then $x_{n} \preceq x$ for all $n \geq 0$;

if a nonincreasing sequence $\left\{x_{n}\right\} \rightarrow x$ in $(X, d)$, then $x_{n} \succeq x$ for all $n \geq 0$.

Regularity the mapping with define by $f: X \rightarrow X$ need not be nondecreasing. Alias, it is sufficient to consider monotone, that is, $f$ may be nondecreasing or nonincreasing.

Some notations are defined as follows.

(1) Let $\mathcal{F}$ be the set of functions $\xi:[0, \infty) \rightarrow[0, \infty)$ satisfying the condition $\xi(t)=0$ if and only if $t=0$.

(2) We denote by $\Psi$ the set of functions $\psi \in \mathscr{F}$ such that $\psi$ is continuous and nondecreasing.

(3) We denote by $\Phi$ the set of functions $\phi \in \mathcal{F}$ such that $\phi$ is lower semicontinuous.

(4) Let $(X, \preceq, d)$ be a metric ordered metric space and $f: X \rightarrow X$ be mappings for all distinct comparable points $x, y \in X$,

$$
M(x, y)=\max \left\{d(x, y), d(x, f x), d(f x, f y), \frac{d(x, f x) d(y, f y)}{d(x, y)}\right\},
$$

$N_{L}(x, y)=L \min \{d(x, f y), d(x, f x), d(y, f y), d(y, f x)\}$ such that $L \geq 0$.

\section{FiXed POINT THEOREM}

Definition 4 ([6]). $F:[0, \infty)^{2} \rightarrow \mathbb{R}$ is called $C$-class function if it is continuous and satisfies following axioms:

(1) $F(s, t) \leq s$;

(2) $F(s, t)=s$ implies that either $s=0$ or $t=0$;

for all $s, t \in[0, \infty)$.

We denote the set of $C$-class functions by $\mathcal{C}$. It is clear that if $F \in \mathcal{C}$ then $F(0,0)=$ 0 . 
Theorem 2. Let $(X, \preceq, d)$ be a partially ordered Hausdorff and complete RMS. Let $f: X \rightarrow X$ be a monotone mapping satisfying for all distinct points $x, y \in X$ with $x \preceq y$,

$$
\psi(d(f x, f y)) \leq F(\psi(M(x, y)), \phi(M(x, y)))+N_{L}(x, y)
$$

such that $\psi \in \Psi, \phi \in \Phi, F \in \mathcal{C}$. Also, suppose that either

(1) C1) $f$ is continuous or

(2) $C 2)(X, \preceq, d)$ be regular.

If there exists $x_{0} \in X$ with $x_{0} \preceq f x_{0}$ or $x_{0} \succeq f x_{0}$, then $f$ has a fixed point in $X$. Moreover, if $(X, \preceq)$ has the property (1.4) (or (1.5)), then the fixed point is unique.

Proof. Let $x_{0} \in X$ such that $x_{0} \preceq f x_{0}$ or $x_{0} \succeq f x_{0}$. Define the sequence $\left\{x_{n}\right\}$ by $x_{n}=f x_{n-1}$ for $n \geq 1$. If there there exists $n_{0}$ such that $x_{n_{0}}=x_{n_{0}+1}$. Then $f$ has a fixed point. Suppose that $x_{n} \neq x_{n+1}$ for all $n \in \mathbb{N}$. In this case, we give the proof step by step.

Step 1: The sequence $\left\{x_{n}\right\}$ defined by $x_{n}=f x_{n-1}$ for $n \geq 1$ is monotone. In fact, since $f$ is monotone, we have

$$
x_{0} \preceq x_{1}=f x_{0} \preceq x_{2}=f^{2} x_{0} \preceq \cdots \preceq x_{n}=f^{n} x_{0} \preceq \cdots
$$

or

$$
x_{0} \succeq x_{1}=f x_{0} \succeq x_{2}=f^{2} x_{0} \succeq \cdots \succeq x_{n}=f^{n} x_{0} \succeq \cdots
$$

Step 2: We claim that $d\left(x_{n}, x_{n+1}\right) \rightarrow 0$. Indeed, since $\left\{x_{n}\right\}$ is a monotone sequence, from contractive inequality, we have

$$
\begin{aligned}
\psi\left(d\left(x_{n}, x_{n+1}\right)\right) & =\psi\left(d\left(f x_{n-1}, f x_{n}\right)\right) \\
& \leq F\left(\psi\left(M\left(x_{n-1}, x_{n}\right)\right), \phi\left(M\left(\left(x_{n-1}, x_{n}\right)\right)\right)\right)+N_{L}\left(\left(x_{n-1}, x_{n}\right)\right) \\
& \leq \psi\left(M\left(x_{n-1}, x_{n}\right)\right)+N_{L}\left(\left(x_{n-1}, x_{n}\right)\right)
\end{aligned}
$$

where

$$
\begin{gathered}
M\left(x_{n-1}, x_{n}\right)= \\
\max \left\{d\left(x_{n-1}, x_{n}\right), d\left(x_{n-1}, f x_{n-1}\right), d\left(x_{n}, x_{n+1}\right), \frac{d\left(x_{n-1}, f x_{n-1}\right) d\left(x_{n}, f x_{n}\right)}{d\left(x_{n-1}, x_{n}\right)}\right\} \\
=\max \left\{d\left(x_{n-1}, x_{n}\right), d\left(x_{n}, x_{n+1}\right)\right\}
\end{gathered}
$$

and

$$
\begin{aligned}
N_{L}\left(x_{n-1}, x_{n}\right) & =L \min \left\{d\left(x_{n-1}, x_{n+1}\right), d\left(x_{n-1}, x_{n}\right), d\left(x_{n}, x_{n+1}\right), d\left(x_{n}, x_{n}\right)\right\} \\
& =0 .
\end{aligned}
$$

Thus, from (3.5), it is clear that $\left\{d\left(x_{n}, x_{n+1}\right)\right\}$ is a monotone nonincreasing sequence of non-negative real numbers. Thus, there exists $r \geq 0$ such that $d\left(x_{n}, x_{n+1}\right) \rightarrow r$ as $n \rightarrow \infty$. 
Taking limit as $n \rightarrow \infty$ in (3.4), we have

$$
\begin{aligned}
\lim _{n \rightarrow \infty} \psi\left(d\left(x_{n}, x_{n+1}\right)\right) & \leq \lim _{n \rightarrow \infty} F\left(\psi\left(M\left(x_{n-1}, x_{n}\right)\right), \phi\left(M\left(\left(x_{n-1}, x_{n}\right)\right)\right)\right) \\
& \leq \lim _{n \rightarrow \infty} \psi\left(M\left(x_{n-1}, x_{n}\right)\right)
\end{aligned}
$$

and

$$
\psi(r) \leq F(\psi(r), \phi(r)) \leq \psi(r)
$$

this implies that $F(\psi(r), \phi(r))=\psi(r)$. From the definiton of $F$ function, $\psi(r)=$ 0 or $\phi(r)=0$. So, we hold that

$$
\lim _{n \rightarrow \infty} d\left(x_{n}, x_{n+1}\right)=0 .
$$

Step 3: We claim that $d\left(x_{n}, x_{n+2}\right) \rightarrow 0$. Indeed, from contractive inequality we have

$$
\begin{aligned}
\psi\left(d\left(x_{n}, x_{n+2}\right)\right)= & \psi\left(d\left(f x_{n-1}, f x_{n+1}\right)\right) \\
\leq & F\left(\psi\left(M\left(x_{n-1}, x_{n+1}\right)\right), \phi\left(M\left(\left(x_{n-1}, x_{n+1}\right)\right)\right)\right) \\
& +N_{L}\left(\left(x_{n-1}, x_{n+1}\right)\right) \\
\leq & \psi\left(M\left(x_{n-1}, x_{n+1}\right)\right)+N_{L}\left(\left(x_{n-1}, x_{n+1}\right)\right)
\end{aligned}
$$

where

$$
\begin{aligned}
& M\left(x_{n-1}, x_{n+1}\right)=\max \left\{\begin{array}{c}
d\left(x_{n-1}, x_{n+1}\right), d\left(x_{n-1}, f x_{n-1}\right), d\left(x_{n}, x_{n+2}\right), \\
\frac{d\left(x_{n-1}, f x_{n-1}\right) d\left(x_{n+1}, f x_{n+1}\right)}{d\left(x_{n-1}, x_{n+1}\right)}
\end{array}\right\} \\
& =\max \left\{d\left(x_{n-1}, x_{n+1}\right), d\left(x_{n-1}, x_{n}\right), d\left(x_{n}, x_{n+2}\right), \frac{d\left(x_{n-1}, x_{n}\right) d\left(x_{n+1}, x_{n+2}\right)}{d\left(x_{n-1}, x_{n+1}\right)}\right\} .
\end{aligned}
$$

As a convenience, let

$$
a_{n}=d\left(x_{n-1}, x_{n+1}\right), b_{n}=d\left(x_{n-1}, x_{n+1}\right), \text { for all } n \geq 1 .
$$

Therefore, we obtain

$$
M\left(x_{n-1}, x_{n+1}\right)=\max \left\{a_{n}, b_{n}, a_{n+1}, \frac{b_{n} b_{n+2}}{a_{n}}\right\} .
$$

If

$$
M\left(x_{n-1}, x_{n+1}\right)=b_{n} \text { or } M\left(x_{n-1}, x_{n+1}\right)=\frac{b_{n} b_{n+2}}{a_{n}}
$$

then thanks to properties of $\psi$ function, we have $d\left(x_{n}, x_{n+2}\right) \rightarrow 0$ as $n \rightarrow \infty$. If

$$
M\left(x_{n-1}, x_{n+1}\right)=a_{n} \text { or } M\left(x_{n-1}, x_{n+1}\right)=a_{n+1}
$$

then, we see that $\left\{d\left(x_{n}, x_{n+2}\right)\right\}$ is a monotone nonincreasing sequence of non-negative real numbers. As shown above, we get that $d\left(x_{n}, x_{n+2}\right) \rightarrow 0$ as $n \rightarrow \infty$. As a result, for all cases in (3.9), we have

$$
\lim _{n \rightarrow \infty} d\left(x_{n}, x_{n+2}\right)=0 .
$$


Step 4: We claim that $f$ has a periodic point. Assume that $f$ has no periodic point; then $\left\{x_{n}\right\}$ is a sequence of distinct points, that is, $x_{n} \neq x_{m}$ for all $m \neq n$. In this case we will get that $\left\{x_{n}\right\}$ is a g.m.s Cauchy sequence. If not, then there exists some $\epsilon>0$ for which we can find two sequences $\left\{x_{m(k)}\right\}$ and $\left\{x_{n(k)}\right\}$ of $\left\{x_{n}\right\}, m(k)>n(k)>k$ for each $k \geq 0$ such that

$$
d\left(x_{m(k)}, x_{n(k)}\right) \geq \epsilon \text { and } d\left(x_{m(k)-1}, x_{n(k)}\right)<\epsilon .
$$

Note that $\left\{x_{n}\right\}$ is a sequence of distinct points, then thanks to rectangular inequality, we have

$$
\begin{aligned}
\epsilon & \leq d\left(x_{m(k)}, x_{n(k)}\right) \\
& \leq d\left(x_{m(k)}, x_{m(k)-2}\right)+d\left(x_{m(k)-2}, x_{m(k)-1}\right)+d\left(x_{m(k)-1}, x_{n(k)}\right) \\
& \leq d\left(x_{m(k)}, x_{m(k)-2}\right)+d\left(x_{m(k)-2}, x_{m(k)-1}\right)+\epsilon .
\end{aligned}
$$

Thus,

$$
\lim _{k \rightarrow \infty} d\left(x_{m(k)}, x_{n(k)}\right)=\epsilon
$$

Also, we get

$$
\begin{aligned}
\psi\left(d\left(x_{m(k)}, x_{n(k)}\right)\right) \leq & F\left(\psi\left(M\left(x_{m(k)-1}, x_{n(k)-1}\right)\right), \phi\left(M\left(x_{m(k)-1}, x_{n(k)-1}\right)\right)\right) \\
& +N_{L}\left(x_{m(k)-1}, x_{n(k)-1}\right)
\end{aligned}
$$

where

$$
\max \left\{\begin{array}{c}
M\left(x_{m(k)-1}, x_{n(k)-1}\right)= \\
d\left(x_{m(k)-1}, x_{n(k)-1}\right), d\left(x_{m(k)-1}, x_{m(k)}\right), d\left(x_{m(k)}, x_{n(k)}\right) \\
\frac{d\left(x_{m(k)-1}, x_{m(k)}\right) d\left(x_{n(k)-1}, x_{n(k)}\right)}{d\left(x_{m(k)-1}, x_{n(k)-1}\right)}
\end{array}\right\}
$$

and

$$
\begin{gathered}
N_{L}\left(x_{m(k)-1}, x_{n(k)-1}\right)= \\
L \min \left\{d\left(x_{m(k)-1}, x_{n(k)}\right), d\left(x_{m(k)-1}, x_{m(k)}\right), d\left(x_{n(k)-1}, x_{n(k)}\right), d\left(x_{n(k)-1}, x_{m(k)}\right)\right\} .
\end{gathered}
$$

Let $n \rightarrow \infty$ in (3), we have

$$
\psi(\epsilon) \leq F(\psi(\epsilon), \phi(\epsilon)) \leq \psi(\epsilon) .
$$

Thus we have $F(\psi(\epsilon), \phi(\epsilon))=\psi(\epsilon)$. From definition of $F$ class function and $\psi$ function we get that $\epsilon=0$. This is a contradiction. Hence, $\left\{x_{n}\right\}$ is a g.m.s Cauchy sequence. Thus there exists $u \in X$ such that the sequence $\left\{x_{n}\right\}$ converges to $u$. In here, we have two cases:

- Let $\mathrm{C} 1$ ) holds, that is, $f$ be continuous, then we have

$$
u=\lim _{n \rightarrow \infty} x_{n}=\lim _{n \rightarrow \infty} f x_{n-1}=f \lim _{n \rightarrow \infty} x_{n-1}=f u .
$$


- Let C2) holds, that is, $(X, \preceq, d)$ be regular. From (3.2) and (3.3), it is clear that the sequence $\left\{x_{n}\right\}$ is a monotone sequence. Thus, by the regularity of $X$, we have $x_{n} \succeq u$ or $x_{n} \preceq u$ for all $n \geq 1$. This means that for each $n \geq 1 x_{n}$ and $u$ are comparable elements in the sense of partially order " $\preceq$ ". Note that $f$ is a monotone mapping, then for each $n \geq 1 f x_{n}$ and $f u$ are comparable elements. This is lets to use contractive inequality (3.1);

$$
\begin{aligned}
\psi\left(d\left(f x_{n}, f u\right)\right) & =\psi\left(d\left(f x_{n-1}, f u\right)\right) \\
& \leq F\left(\psi\left(M\left(x_{n-1}, u\right)\right), \phi\left(M\left(\left(x_{n-1}, u\right)\right)\right)\right)+N_{L}\left(\left(x_{n-1}, u\right)\right) \\
& \leq \psi\left(M\left(x_{n-1}, u\right)\right)+N_{L}\left(\left(x_{n-1}, u\right)\right)
\end{aligned}
$$

where

$$
\begin{gathered}
M\left(x_{n-1}, u\right)= \\
\max \left\{d\left(x_{n-1}, u\right), d\left(x_{n-1}, x_{n}\right), d\left(f x_{n-1}, f u\right), \frac{d\left(x_{n-1}, x_{n}\right) d(u, f u)}{d\left(x_{n-1}, u\right)}\right\}
\end{gathered}
$$

and

$$
N_{L}\left(x_{n-1}, u\right)=L \min \left\{d\left(x_{n-1}, f u\right), d\left(x_{n-1}, x_{n}\right), d(u, f u), d\left(u, x_{n}\right)\right\} .
$$

Let $n \rightarrow \infty$ in (3.13) and (3.14), we obtain

$$
\lim _{n \rightarrow \infty} M\left(x_{n-1}, u\right)=d(u, f u) \text { and } \lim _{n \rightarrow \infty} N_{L}\left(x_{n-1}, u\right)=0 .
$$

Also, let $n \rightarrow \infty$ in (3.12) and use (3.15), we have

$$
\psi(d(u, f u)) \leq F(\psi(d(u, f u)), \phi(d(u, f u))) \leq \psi(d(u, f u))
$$

this implies $F(\psi(d(u, f u)), \phi(d(u, f u)))=\psi(d(u, f u))$. From the definition of $F$ function $\psi(d(u, f u))=0$ or $\phi(d(u, f u))=0$. We arrive at

$$
d(u, f u)=0 \text { and } f u=u .
$$

Note that (3.11) and (3.17) are contradiction with assumption that $f$ has no periodic point. Consequently, $f$ has a periodic point, that is, there exists $a \in X$ such that $a=f^{p} a$.

Step 5: We claim that $f$ has a fixed point. Note that there exists $a \in X$ such that $a=f^{p} a$. It is clear that $a \in X$ is a fixed point $f$ for $p=1$. We will prove that $\vartheta=f^{p-1} a$ is a fixed point of $f$ in case of $p>1$. If possible, assume the contrary, that is, let $f^{p-1} a \neq f^{p} a$. Thus, from the contractive inequality, we get

$$
\begin{aligned}
& \psi(d(a, f a))=\psi\left(d\left(f^{p} a, f^{p+1} a\right)\right) \\
& \quad \leq F\left(\psi\left(M\left(f^{p-1} a, f^{p} a\right)\right), \phi\left(M\left(f^{p-1} a, f^{p} a\right)\right)\right)+N_{L}\left(f^{p-1} a, f^{p} a\right)
\end{aligned}
$$


where

$$
\begin{aligned}
M\left(f^{p-1} a, f^{p} a\right) & =\max \left\{\begin{array}{c}
d\left(f^{p-1} a, f^{p} a\right), d\left(f^{p-1} a, f^{p} a\right), d\left(f^{p} a, f^{p+1} a\right), \\
\frac{d\left(f^{p-1} a, f^{p} a\right) d\left(f^{p} a, f^{p+1} a\right)}{d\left(f^{p-1} a, f^{p} a\right)}
\end{array}\right\} \\
& =\max \left\{d\left(f^{p-1} a, f^{p} a\right), d\left(f^{p} a, f^{p+1} a\right)\right\} .
\end{aligned}
$$

Note that $\left\{d\left(x_{n}, x_{n+1}\right)\right\}$ is a monotone nonincreasing sequence of non-negative real numbers, then we get that

$$
M\left(f^{p-1} a, f^{p} a\right)=d\left(f^{p-1} a, f^{p} a\right) \text { and } N_{L}\left(f^{p-1} a, f^{p} a\right)=0 .
$$

Consider (3.18) and definition of $F$ class function, then we have

$$
\begin{aligned}
\psi(d(a, f a)) & =\psi\left(d\left(f^{p} a, f^{p+1} a\right)\right) \\
& \leq F\left(\psi\left(d\left(f^{p-1} a, f^{p} a\right)\right), \phi\left(d\left(f^{p-1} a, f^{p} a\right)\right)\right) \\
& \leq \psi\left(d\left(f^{p-1} a, f^{p} a\right)\right) .
\end{aligned}
$$

From the definition of $\psi$ function, we have $d(a, f a)<d\left(f^{p-1} a, f^{p} a\right)$. Continuing the similar process, we get the following contradiction

$$
d(a, f a)<d\left(f^{p-1} a, f^{p} a\right) \leq d\left(f^{p-2} a, f^{p-1} a\right)<\cdots \leq d(a, f a) .
$$

Hence, the assumption that $\vartheta=f^{p-1} a$ is not a fixed point of $f$ is not true. Consequently, $f$ has a fixed point.

Step 6: The fixed point is unique. Now, we show that this fixed point is unique. If possible, let $\vartheta, \vartheta^{\prime} \in X$ be fixed point of $f$. We distinguish two cases:

- Case 1: If $\vartheta$ is comparable to $\vartheta^{\prime}$ then $f \vartheta=\vartheta$ is comparable to $f \vartheta^{\prime}=\vartheta^{\prime}$. Note that

$$
\begin{aligned}
\psi\left(d\left(\vartheta, \vartheta^{\prime}\right)\right) & =\psi\left(d\left(f \vartheta, f \vartheta^{\prime}\right)\right) \\
& \leq F\left(\psi\left(M\left(\vartheta, \vartheta^{\prime}\right)\right), \phi\left(M\left(\vartheta, \vartheta^{\prime}\right)\right)\right)+N_{L}\left(\vartheta, \vartheta^{\prime}\right) \\
& \leq \psi\left(M\left(\vartheta, \vartheta^{\prime}\right)\right)+N_{L}\left(\vartheta, \vartheta^{\prime}\right)
\end{aligned}
$$

where

$$
M\left(\vartheta, \vartheta^{\prime}\right)=d\left(\vartheta, \vartheta^{\prime}\right) \text { and } N_{L}\left(\vartheta, \vartheta^{\prime}\right)=0 .
$$

Thus, from (3.19), we have

$\psi\left(d\left(\vartheta, \vartheta^{\prime}\right)\right) \leq F\left(\psi\left(d\left(\vartheta, \vartheta^{\prime}\right)\right), \phi\left(d\left(\vartheta, \vartheta^{\prime}\right)\right)\right) \leq \psi\left(d\left(\vartheta, \vartheta^{\prime}\right)\right)$.

From definition of $F$ function and property of $\psi, \phi$ functions, we obtain that $\vartheta=\vartheta^{\prime}$.

- Case 2: If $\vartheta$ is not comparable to $\vartheta^{\prime}$, then there exists an element $z \in X$ such that $z$ comparable to $\vartheta$ and $\vartheta^{\prime}$. Without loss of generalty, we choose $z \preceq \vartheta$. Let define a sequence $\left\{z_{n}\right\}$ by

$$
z_{0}=z \text { and } z_{n}=f z_{n-1}, \text { for all } n \geq 1 .
$$


Note that $f$ is monotone, then we have

$$
f^{n} z \preceq f^{n} \vartheta=\vartheta \text { or } f^{n} z \succeq f^{n} \vartheta=\vartheta
$$

that is, we have

$$
z_{n} \preceq \vartheta \text { or } z_{n} \succeq \vartheta .
$$

If there exists $n_{0}$ such that $\vartheta=z_{n_{0}}$, then $z_{n}=f z_{n-1}=f \vartheta=\vartheta$ for all $n \geq n_{0}-1$. So $\lim _{n \rightarrow \infty} z_{n}=\vartheta$. Analogously, we get $\lim _{n \rightarrow \infty} z_{n}=\vartheta^{\prime}$. From the uniqueness of the limit $\vartheta=\vartheta^{\prime}$.

If $\vartheta \neq z_{n}$ for all $n=0,1,2, \cdots$, then from (3.1), we have

$$
\begin{aligned}
\psi\left(d\left(\vartheta, z_{n}\right)\right) & =\psi\left(d\left(f \vartheta, f z_{n-1}\right)\right) \\
& \leq F\left(\psi\left(M\left(\vartheta, z_{n-1}\right)\right), \phi\left(M\left(\vartheta, z_{n-1}\right)\right)\right)+N_{L}\left(\vartheta, z_{n-1}\right)
\end{aligned}
$$

where

$$
M\left(\vartheta, z_{n-1}\right)=\max \left\{d\left(\vartheta, z_{n-1}\right), d\left(\vartheta, z_{n}\right)\right\} \text { and } N_{L}\left(\vartheta, z_{n-1}\right)=0 .
$$

Considering (3.23) with (3.1), we obtain

$$
\psi\left(d\left(\vartheta, z_{n}\right)\right) \leq F\left(\psi\left(M\left(\vartheta, z_{n-1}\right)\right), \phi\left(M\left(\vartheta, z_{n-1}\right)\right)\right) \leq \psi\left(M\left(\vartheta, z_{n-1}\right)\right) .
$$

This is implies that $\left\{d\left(\vartheta, z_{n}\right)\right\}$ is a monotone decreasing sequences of nonnegative real numbers. Thus, there exists $\lambda \geq 0$ such that $\left\{d\left(\vartheta, z_{n}\right)\right\}$ converges to $\lambda$ as $n \rightarrow \infty$. Letting $n \rightarrow \infty$ in (3.24), we have

$$
\psi(\lambda) \leq F(\psi(\lambda), \phi(\lambda)) \leq \psi(\lambda)
$$

and this implies that $\lambda=0$, that is,

$$
\lim _{n \rightarrow \infty} d\left(\vartheta, z_{n}\right)=0
$$

Analogously, we have

$$
\lim _{n \rightarrow \infty} d\left(\vartheta^{\prime}, z_{n}\right)=0
$$

From the quadrilateral inequality of rectangular metric function we have

$$
d\left(\vartheta, \vartheta^{\prime}\right) \leq d\left(\vartheta, z_{n}\right)+d\left(z_{n}, \vartheta^{\prime}\right)+d\left(\vartheta^{\prime}, \vartheta^{\prime}\right)
$$

take $n \rightarrow \infty$ in (3.26), we hold that $\vartheta=\vartheta^{\prime}$, that is fixed point is unique.

The following theorem obtained by picking out $F(s, t)=s-t$.

Theorem 3. Let $(X, \preceq, d)$ be a partially ordered Hausdorff and complete RMS. Let $f: X \rightarrow X$ be a monotone mapping satisfying for all distinct points $x, y \in X$ with $x \preceq y$,

$$
\psi(d(f x, f y)) \leq \psi(M(x, y))-\phi(M(x, y))+N_{L}(x, y)
$$

such that $\psi \in \Psi, \phi \in \Phi$. Also, suppose either 
(1) C1) $f$ is continuous or

(2) $C 2)(X, \preceq, d)$ be regular.

If there exists $x_{0} \in X$ with $x_{0} \preceq f x_{0}$ or $x_{0} \succeq f x_{0}$, then $f$ has a fixed point in $X$. Moreover, if $(X, \preceq)$ has the property (1.4) (or (1.5)), then the fixed point is unique.

Example 1. Take the $X=\{0,1,2,3\}$ and define usual order " $\leq$ " on $X$. Define a function $d: X \times X \rightarrow[0, \infty)$ as following;

$$
\begin{gathered}
d(1,3)=d(3,1)=4 \\
d(1,2)=d(2,1)=2 \\
d(2,3)=d(3,2)=1 \\
d(x, y)=|x-y|, \text { for other cases. }
\end{gathered}
$$

Note that $d(1,3)>d(1,2)+d(2,3)$, then $d$ is not a (usual) metric on $X$. On the other hand $d$ is a generalized (rectangular) metric on $X$. Define $f: X \rightarrow X$ by

$$
f=\left(\begin{array}{l}
0123 \\
0231
\end{array}\right) \text {. }
$$

Case 1: Let $(x, y)=(1,3) \Longrightarrow d(f 1, f 3)=d(2,1)=2$ and $M(1,3)=4$, $N_{L}(1,3)=0$.

Case 2: Let $(x, y)=(1,2) \Longrightarrow d(f 1, f 2)=d(2,3)=1$ and $M(1,2)=4$, $N_{L}(1,2)=0$.

Case 3: Let $(x, y)=(2,3) \Longrightarrow d(f 2, f 3)=d(3,1)=4$ and $M(2,3)=4$, $N_{L}(2,3)=0$.

Case 4: Let $(x, y)=(1,0) \Longrightarrow d(f 1, f 0)=d(2,0)=2$ and $M(1,2)=2$, $N_{L}(1,2)=0$.

Case 5: Let $(x, y)=(2,0) \Longrightarrow d(f 2, f 0)=d(3,0)=3$ and $M(2,0)=3$, $N_{L}(2,0)=0$.

Case 6: Let $(x, y)=(3,0) \Longrightarrow d(f 3, f 0)=d(1,0)=1$ and $M(3,0)=4$, $N_{L}(3,0)=0$.

For all cases there exists $\psi \in \Psi, \phi \in \Phi, F \in \mathcal{C}$. One of the choices:

$$
\begin{gathered}
F:[0, \infty)^{2} \rightarrow \mathbb{R}, \text { by } F(s, t)=s-t \\
\psi:[0, \infty) \rightarrow[0, \infty) \text { is any continuous and nondecreasing, } \\
\phi:[0, \infty) \rightarrow[0, \infty) \text { by } \phi(\delta)=0 .
\end{gathered}
$$

Therefore, all conditions of Theorem 2 are satisfied. Hence, $f$ has a unique fixed point. In fact $u=0$ is the unique fixed point of $f$. 


\section{SOME AUXILIARY RESULTS}

In this section we aim to give some results that obtained from our main theorem.

Example 2. The following functions $F:[0, \infty)^{2} \rightarrow \mathbb{R}$ are elements of $\mathcal{C}$.

(1) $F(s, t)=k s, 0<k<1$,

(2) $F(s, t)=s-t$,

(3) $F(s, t)=\frac{s}{(1+t)^{r}}, r \in(0, \infty)$.

Theorem 4 (Lakzian and Samet [12]). Let $(X, d)$ be a Hausdorff and complete g.m.s and let $T: X \rightarrow X$ be a self-mapping satisfying

$$
\psi(d(T x, T y)) \leq \psi(d(x, y))-\varphi(d(x, y))
$$

for all $x, y \in X$, where $\psi \in \Psi$ and $\varphi:[0, \infty) \rightarrow[0, \infty)$ is continuous and $\varphi(t)=0$ if and only if $t=0$. Then $T$ has a unique fixed point.

In 2011, Luong \& Thuan [13] introduced a generalized rational contractive inequality to obtain a fixed point result in partially ordered metric space. The following theorem extends Theorem 2.1 of [13] from partially ordered metric space to partially ordered rectangular metric space.

Theorem 5. Let $(X, \preceq)$ be a partially ordered Hausdorff and complete RMS. Let $T: X \rightarrow X$ be an nondecreasing mapping satisfying the following inequality

$$
d(T x, T y) \leq M(x, y)-\varphi(M(x, y)), \text { for all } x, y \in X \text { with } x \succeq y, x \neq y
$$
where

(1) $\phi:[0, \infty) \rightarrow[0, \infty)$ is a lower semi-continuous function with $\phi(t)=0 \Leftrightarrow$ $t=0$

(2) $M(x, y)=\max \left\{\frac{d(x, T x) d(y, T y)}{d(x, y)}, d(x, y)\right\}$.

Also, assume either $T$ is continuous or $X$ has the property (1.3). If there exists $x_{0} \preceq T x_{0}$, then $T$ has a fixed point. Moreover, if $X$ has the property (1.5), then the fixed point is unique.

Arshad et.al [1], by introducing the concept of almost Jaggi contraction, they gave some fixed point theorems for rational contractions in partially ordered metric spaces. The following theorem extends Theorem 2 of [1] from partially ordered metric space to partially ordered rectangular metric space.

Definition 5. Let $(X, \preceq, d)$ be a partially ordered rectangular metric space. A self mapping $f$ on $X$ is called an almost Jaggi contraction if it satisfies the following condition:

$$
d(f x, f y) \leq \alpha \frac{d(x, f x) d(y, f y)}{d(x, y)}+\beta d(x, y)+L \min \{d(x, f y), d(y, f x)\},
$$

for any distinct $x, y \in X$ with $x \preceq y$, where $L \geq 0$ and $\alpha, \beta \in[0,1)$ with $\alpha+\beta<1$. 
Theorem 6. Let $(X, \preceq, d)$ be a partially ordered Hausdorff and complete RMS. Suppose that a self-mapping $f$ is an almost Jaggi contraction, continuous and nondecreasing. Suppose there exists $x_{0} \in X$ with $x_{0} \preceq f x_{0}$. Then $f$ has a unique fixed point.

Mustafa et. al.[14] used the definition of almost contraction to make a discussion on generalized almost contractions via rational expressions in partially ordered metric space. The following theorem extends Theorem 3 of [14] from partially ordered metric space to partially ordered rectangular metric space.

Theorem 7. Let $(X, \preceq)$ be a partially ordered Hausdorff and complete RMS. Let $f: X \rightarrow X$ be a non-decreasing mapping which satisfies the inequality

$$
\begin{gathered}
\phi(d(T x, T y)) \leq \\
\phi(M(x, y))-\psi(M(x, y))+L \min \{d(x, f y), d(x, f x), d(y, f y), d(y, f x)\},
\end{gathered}
$$

for all distinct points $x, y \in X$ with $y \leq x$ where $\phi \in \Phi, \psi \in \Psi, L \geq 0$ and

$$
M(x, y)=\max \left\{\frac{d(x, f x) d(y, f y)}{d(x, y)}, d(x, y)\right\} .
$$

Also, assume either $f$ is continuous or $X$ has the feature (1.3). If there exists $x_{0} \in X$ such that $x_{0} \preceq f x_{0}$, then $f$ has a fixed point. Moreover, if $X$ has the feature (1.5), then the fixed point is unique.

\section{Applications}

Let $\Lambda$ be the set of functions $\xi:[0,+\infty) \rightarrow[0,+\infty)$ such that

(1) $\xi$ is Lebesgue integrable mapping on each compact subset of $[0,+\infty)$;

(2) $\int_{0}^{\varepsilon} \xi(s) d s>0$ for every $\varepsilon>0$.

For this class of functions, we can express the following results.

Theorem 8. Let $(X, d)$ be a Hausdorff and complete RMS, and let $f$ be a self-map satisfying

$$
\int_{0}^{d(f x, f y)} \xi_{1}(s) d s \leq F\left(\int_{0}^{M(x, y)} \xi_{1}(s) d s, \int_{0}^{M(x, y)} \xi_{2}(s) d s\right)+N_{L}(x, y)
$$

for all $x, y \in X$ and $\xi_{1}, \xi_{2} \in \Lambda, F \in \mathcal{C}$ and

$$
M(x, y)=\max \left\{d(x, y), d(x, f x), d(f x, f y), \frac{d(x, f x) d(y, f y)}{d(x, y)}\right\},
$$

$N_{L}(x, y)=L \min \{d(x, f y), d(x, f x), d(y, f y), d(y, f x)\}$ such that $L \geq 0$.

Then $f$ has a unique fixed point $\vartheta \in X$.

Proof. Let $\psi(s)=\int_{0}^{s} \xi_{1}(v) d v$ and $\phi(s)=\int_{0}^{s} \xi_{2}(v) d v$. Then $\psi \in \Psi, \phi \in \Phi$, and beside, the function $\psi$ is nondecreasing. By Theorem 2, $T$ has a unique fixed point. 
Taking $\xi_{2}(s)=(1-k) \xi_{1}(s)$ and $F(s, t)=s-t$ in Theorem 8, we obtain the following statement.

Corollary 1. Let $(X, d)$ be a Hausdorff and complete RMS, and let $f$ be a selfmap satisfying

$$
\begin{aligned}
& \int_{0}^{d(f x, f y)} \xi_{1}(s) d s \leq
\end{aligned}
$$

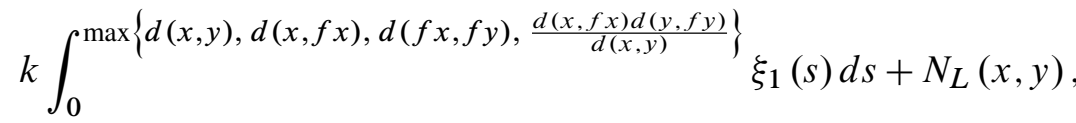

for all $x, y \in X$ and $\xi_{1}, \xi_{2} \in \Lambda, F \in \mathcal{C}$.

Then $f$ has a unique fixed point $\vartheta \in X$.

Taking $F(s, t)=s-t$ in Theorem 8 , we have the following statement.

Corollary 2. Let $(X, d)$ be a Hausdorff and complete RMS, and let $f$ be a selfmap satisfying

$$
\int_{0}^{d(f x, f y)} \xi_{1}(s) d s \leq \int_{0}^{M(x, y)} \xi_{1}(s) d s-\int_{0}^{M(x, y)} \xi_{2}(s) d s+N_{L}(x, y),
$$

for all $x, y \in X$ and $\xi_{1}, \xi_{2} \in \Lambda, F \in \mathcal{C}$ and

$$
M(x, y)=\max \left\{d(x, y), d(x, f x), d(f x, f y), \frac{d(x, f x) d(y, f y)}{d(x, y)}\right\},
$$

$N_{L}(x, y)=L \min \{d(x, f y), d(x, f x), d(y, f y), d(y, f x)\}$ such that $L \geq 0$.

Then $f$ has a unique fixed point $\vartheta \in X$.

Taking $F(s, t)=k s, 0<k<1$ and $L=0$ in Theorem 8, we get the following statement.

Corollary 3. Let $(X, d)$ be a Hausdorff and complete RMS, and let $f$ be a selfmap satisfying

$$
\int_{0}^{d(f x, f y)} \xi_{1}(s) d s \leq k \int_{0}^{\max \left\{d(x, y), d(x, f x), d(f x, f y), \frac{d(x, f x) d(y, f y)}{d(x, y)}\right\}} \xi_{1}(s) d s,
$$

for all $x, y \in X$ and $\xi_{1}, \xi_{2} \in \Lambda, F \in \mathcal{C}$.

Then $f$ has a unique fixed point $\vartheta \in X$.

\section{CONCLUSiON}

Our theorems and corolaries which include the corresponding results studied in Luong \& Thuan (2011), Arshad et al. (2013), Mustafa et al. (2014), Kir \& Kiziltunc (2015) as special cases fundamentally improve and generalize the results of ([3], [7], [10], [17], [19], [5], [4], [18], [2], [12], [16], [15], [8], [9]) in the following sense. 
(i) Rectangular metric space has substituted the sum at the righthand side of the triangle inequality by a three-term expression.

( $i$ i ) Generalized from metric space to rectangular metric space.

\section{REFERENCES}

[1] M. Arshad, E. Karapınar, and J. Ahmad, "Some unique fixed point theorems for rational contractions in partially ordered metric spaces." J. Inequal. Appl., vol. 2013, no. 248, pp. 1-16, 2013, doi: 10.1186/1029-242X-2013-248.

[2] H. Aydi, E. Karapınar, and H. Lakzian, "Fixed point results on a class of generalized metric spaces." Math. Sci., vol. 6, no. 46, pp. 1-6, 2012, doi: 10.1186/2251-7456-6-46.

[3] S. Banach, "Sur les operations dans les ensembles abstraits et leur application aux equations integerales." Fund. Math., vol. 3, no. 1, pp. 133-181, 1922.

[4] A. Branciari, "Fixed point theorem of Banach-Caccioppoli type on a class of generalized metric spaces.” Publ. Math. Debrecen, vol. 57, no. 1, pp. 31-37, 2000.

[5] D. Doric, "Common fixed point theorem for generalized $(\psi, \varphi)$-weak contractions." Appl. Math. Lett., vol. 22, no. 12, pp. 1896-1900, 2009, doi: 10.1016/j.aml.2009.08.001.

[6] Z. Fadail, A. Ahmad, A. Ansari, S. Radenovic, and M. Rajovic, "Some common fixed point results of mappings in $0-\sigma-$ complete metric-like spaces via new function." Appl. Math. Sci., vol. 9, no. 83, pp. 4109-4127, 2015, doi: 10.12988/ams.2015.53192.

[7] M. Geraghty, "On contractive mappings." Proc. Amer. Math. Soc., vol. 40, pp. 604-608, 1973, doi: 10.1090/S0002-9939-1973-0334176-5.

[8] A. Harandi and H. Emami, "Fixed point theorem for contraction type maps in partially ordered metric spaces and application to ordinary differential equations." Nonlinear Anal., vol. 72, no. 5, pp. 2238-2242, 2010, doi: 10.1016/j.na.2009.10.023.

[9] J. Harjani, B. López, and K. Sadarangani, “A fixed point theorem for mappings satisfying a contractive condition of rational type on a partially ordered metric space." Abstr. Appl. Anal., vol. 2010, pp. 1-8, 2010, doi: 10.1155/2010/190701.

[10] D. Jaggi, "Some unique fixed point theorems." Indian J. Pure Appl. Math., vol. 8, no. 2, pp. 223-230, 1977.

[11] M. Kir and H. Kiziltunc, "Some generalized fixed point theorems in the context of ordered metric spaces.” J. Nonlinear Sci. Appl. , vol. 8, no. 5, pp. 529-539, 2015.

[12] H. Lakzian and B. Samet, "Fixed points $(\psi, \phi)$-weakly contractive mappings in generalized metric spaces." Appl. Math. Lett., vol. 25, no. 5, pp. 902-906, 2012, doi: 10.1016/j.aml.2011.10.047.

[13] N. Luong and N. Thuan, "Fixed point theorem for generalized weak contractions satisfying rational expressions in ordered metric spaces." Fixed Point Theory Appl., vol. 2011, no. 46, pp. 1-10, 2011, doi: 10.1186/1687-1812-2011-46.

[14] Z. Mustafa, E. Karapınar, and H. Aydi, "A discussion on generalized almost contractions via rational expressions in partially ordered metric spaces." J. Inequal. Appl., vol. 2014, no. 219, pp. 1-12, 2014, doi: 10.1186/1029-242X-2014-219.

[15] J. Nieto and R. Rodríguez-López, "Contractive mapping theorems in partially ordered sets and applications to ordinary differential equations." Order, vol. 22, no. 3, pp. 223-239, 2005, doi: 10.1007/s11083-005-9018-5.

[16] A. Ran and M. Reurings, "A fixed point theorem in partially ordered sets and some applications to matrix equations." Proc. Amer. Math. Soc., vol. 132, no. 5, pp. 1435-1443, 2004, doi: 10.1090/S0002-9939-03-07220-4.

[17] B. Rhoades, "Some theorems on weakly contractive maps." Nonlinear Anal., vol. 41, no. 4, pp. 2683-2693, 2001, doi: 10.1016/S0362-546X(01)00388-1. 
[18] B. Samet, "Discussion on "A fixed point theorem of Banach-Caccioppoli type on a class of generalized metric spaces" by A. Branciari”.” Publ. Math. Debrecen, vol. 76, no. 4, pp. 493-494, 2010.

[19] Q. Zhang and Y. Song, "Fixed point theory for generalized $\varphi$ - weak contractions." Appl. Math. Lett., vol. 22, no. 1, pp. 75-78, 2009, doi: 10.1016/j.aml.2008.02.007.

Authors' addresses

Mehmet Kir

Sirnak University, Department of Civil Engineering, Faculty of Engineering, 73000 Sirnak, Turkey

E-mail address: mehmetkir04@gmail.com

Mehmet Ali Akturk

Istanbul University, Department of Engineering Sciences, 34320 Istanbul, Turkey

E-mail address: mehmetaliakturk@yandex.com

\section{Hemen Dutta}

Gauhati University, Department of Mathematics, 781014 Guwahati, India

E-mail address: hemen_dutta08@rediffmail.com

\section{Esra Yolacan}

Republic of Turkey Ministry of National Education, 60000 Tokat, Turkey

E-mail address: yolacanesra@gmail.com 